\title{
La Cuarta R: Un Programa Escolar de Prevención de la Violencia en las Relaciones de Pareja en la Adolescencia
}

\section{The Fourth R: A School-Based Adolescent Dating Violence Prevention Program}

\author{
David A.Wolfe ${ }^{1,2}$, Claire V. Crooks ${ }^{1}$ y Raymond Hughes ${ }^{1}$ \\ ${ }^{1} \mathrm{CAMH}$ Centre for Prevention Science, Canada \\ ${ }^{2}$ University of Toronto, Canada
}

\begin{abstract}
Resumen. En este trabajo se presenta un programa de prevención creado para su aplicación en el entorno escolar ( La Cuarta R), que tiene como objetivo la prevención de la violencia contra la pareja en la adolescencia y conductas de riesgo relacionadas. El elemento central de La Cuarta R es un programa de 21 temas curriculares, implementado por profesores que reciben una formación especializada, que trata de promover las relaciones saludables, y cuyo principal objetivo es la violencia, el comportamiento sexual de alto riesgo y el uso de sustancias entre los adolescentes. La Cuarta R se evaluó en 20 escuelas mediante un diseño con asignación aleatoria a la condición experimental o control. Los resultados de la evaluación de este programa de intervención señalan que la inclusión de contenidos curriculares sobre relaciones sociales saludables, así como habilidades para evitar situaciones de violencia física en las relaciones de pareja entre adolescentes, permitía reducir este tipo de violencia, así como incrementaba el uso del condón 2.5 años después.

Palabras clave: adolescencia, escuela, prevención, violencia en las relaciones de noviazgo.
\end{abstract}

\begin{abstract}
This paper presents a school-based primary prevention program (The Fourth $R$ ) to prevent adolescent dating violence, and related risk behaviors. The cornerstone of The Fourth $\mathrm{R}$ is a 21-lesson skillbased curriculum delivered by teachers who receive specialized training, that promotes healthy relationships, and targets violence, high-risk sexual behavior, and substance use among adolescents. The Fourth $\mathrm{R}$ was evaluated in a cluster randomized trial in 20 schools. Results indicated that teaching youth healthy relationships and skills as part of their curriculum reduced physical dating violence, and increased condom use 2.5 years later.

Keywords: adolescence, dating violence, prevention, school.
\end{abstract}

La violencia en las relaciones de pareja durante la adolescencia es un problema con una creciente y preocupante incidencia (Crooks, Jaffe, Wolfe, Hughes y Chiodo, 2011). Aunque históricamente no se le ha dado importancia a este tipo de violencia (debido, en parte, a que los adultos no se han tomado demasiado en serio las relaciones entre adolescentes), hoy en día podemos afirmar que la violencia de pareja que se da entre adolescentes puede tener repercusiones psicológicas y comportamentales muy negativas (Wolfe et al, 2009). En este sentido, este tipo de violencia es uno de los principales precursores de la violencia de pareja en la vida adulta (Centers for Disease Control and Prevention, 2006). Además, se encuentra asociado a conductas peligrosas que pueden poner en riesgo la salud del adolescente (Coker, McKeown, Anderson, Davis, Valois y Huebner, 2002). Así, se ha hallado entre las víctimas una mayor probabilidad de sufrir

Correspondencia: David A. Wolfe, CAMH Centre for Prevention Science, 100 Collip Circle, Suite 100, London, Ontario N6G 4X8, Canada. E-mail: David_Wolfe@camh.netdawolfe@uwo.ca trastornos de estrés post-traumático, depresión (Wolitzky-Taylor et al., 2008), violencia entre iguales y comportamiento suicida (Swahn et al., 2008), abuso de sustancias, trastornos alimenticios y embarazos no deseados (Eaton et al., 2006; Silverman, Raj, Mucci y Hathaway, 2001). Para los adolescentes, la violencia física en las relaciones de pareja, definida como actos que pueden variar desde amenazar con hacer daño hasta dar un puñetazo o golpear con algún objeto (Grunbaum et al., 2001; Lewis y Fremouw, 2001), se produce en un periodo de transición crítico y estresante, que conlleva nuevas presiones y nuevas responsabilidades en el manejo de los conflictos y las emociones en contextos que van más allá del ámbito familiar (Conolly, Furman y Konarski, 2000; Irwin, Burg y Uhler Cart, 2002; Wolfe, Jaffe y Crooks, 2006).

En Estados Unidos, las encuestas nacionales con muestras representativas realizadas desde mediados de los años 90 en relación a la violencia en las relaciones de pareja entre adolescentes, indican que 1 de cada 10 (Lewis y Fremouw, 2001) a 1 de cada 5 (Silverman, Raj, Mucci y Hathaway, 2001) de los adolescentes que 
están en edad de asistir al instituto, son golpeados o abofeteados por su pareja cada año. Además, a diferencia de lo que sucede con la violencia en las relaciones de pareja en muestras de adultos, la mayoría de los estudios realizados con adolescentes encuentran que las tasas de agresión son similares entre chicos y chicas, e incluso ligeramente superiores para las chicas, por razones que aún nos son desconocidas (Odgers y Moretti, 2002; Wolfe, Scott y Crooks, 2005).

Vista la elevada prevalencia de este tipo de violencia entre los adolescentes y la necesidad de reducir este ciclo de violencia por las consecuencias que tiene para la vida adulta, cada vez son más los investigadores que recomiendan encarecidamente educar a los adolescentes para que mantengan relaciones de pareja sanas y eliminen la violencia en sus relaciones, así como los factores de riesgo asociados a esta conducta violenta (Hahn et al., 2007; US Department of Health and Human Services, 2000).

\section{Factores de riesgo asociados a la violencia de pareja en la adolescencia}

Se han identificado numerosos factores de riesgo en relación a la violencia en las relaciones de pareja entre adolescentes, entre los que se incluyen, por ejemplo, factores sociodemográficos tales como el bajo estatus socioeconómico, o la pertenencia a determinadas minoría étnicas, aunque los resultados son inconsistentes (ver, por ejemplo, O'Keefe, 2005). La experiencia o exposición previa a la violencia es otro factor de riesgo. Así, diversas investigaciones han encontrado mayores tasas de violencia en las relaciones de noviazgo entre los jóvenes que han estado expuestos a la violencia doméstica entre sus padres o que han sufrido maltrato infantil (Wolfe, Scott, Wekerle y Pittman, 2001; Wolfe, Crooks, Chiodo y Jaffe, 2009). La aceptación del uso de la violencia es otro importante factor de riesgo de violencia en las relaciones íntimas entre adolescentes (Foshee, Bauman y Linder, 1999). Por otra parte, la influencia de los iguales se ha mostrado como un importante factor de riesgo y como un predictor más potente de violencia en las relaciones de pareja entre adolescentes que la violencia familiar (Arriaga y Foshee, 2004). Por último, la implicación en otras conductas de riesgo se asocia a mayores tasas de violencia de pareja (e.g, Silverman et al., 2001). En un análisis longitudinal de las trayectorias de la violencia infligida por la pareja desde la adolescencia hasta la adultez temprana realizado recientemente, la iniciación sexual temprana y el número de parejas sexuales fueron los predictores más potentes de este tipo de violencia, así como de su continuidad a lo largo del tiempo. Este resultado subraya la importancia de tener en cuenta diferentes conductas de riesgo de forma conjunta (Halpern, Spriggs, Martin y Kupper, 2009).
En cuanto a la relación entre violencia en las relaciones de pareja entre adolescentes y pertenencia a una minoría étnica, los resultados que proporcionan las investigaciones son complejos. Por una parte, algunos estudios realizados en Estados Unidos han mostrado las tasas más elevadas de violencia entre los afroamericanos, siendo las más bajas las obtenidas para los asiáticos y latinos, quedando los caucásicos en una posición intermedia (O'Keefe, 2005). Por otro lado, otros investigadores no han encontrado diferencias en función de la etnia de pertenencia cuando se controlan otras variables (Malik, Sornson y Aneshensel, 1997). En este sentido, aunque algunos resultados puedan sugerir diferencias en función de la etnia de pertenencia, estas diferencias podrían explicarse a partir de factores estructurales tales como, por ejemplo, las tasas de desempleo. Así pues, más que la pertenencia a una determinada etnia, el elemento crítico es la interacción entre etnia y pertenencia a un grupo socialmente desfavorecido (Miller y White, 2003).

\section{La prevención de la violencia en las relaciones de pareja durante la adolescencia desde el entorno escolar}

Como hemos visto, la violencia de pareja entre adolescentes está relacionada con numerosos factores de riesgo individuales, relacionales, familiares y socioculturales. Este hecho impide que sea posible la identificación y tratamiento basados únicamente en factores de riesgo individuales y hace más necesaria, si cabe, la prevención (Wekerle y Wolfe, 1999; Wolfe et al., 2009). Por otra parte, las tasas de prevalencia que se han indicado previamente y las graves consecuencias asociadas a la violencia en las relaciones de pareja entre adolescentes, hacen necesario un enfoque basado en la promoción de las relaciones saludables y la prevención de la violencia de pareja, en lugar de confiar únicamente en la identificación e intervención con los jóvenes que ya cometen o sufren violencia en sus relaciones íntimas (Crooks et al., 2011). Las bajas tasas de búsqueda de ayuda entre los adolescentes que sufren violencia en sus relaciones sentimentales subraya la necesidad de ser proactivos. En este sentido, un estudio ha mostrado que el $60 \%$ de las víctimas y el $79 \%$ de los autores de las agresiones no buscan ayuda para eliminar la violencia en dichas relaciones (Ashley y Foshee, 2005). Además, según el mismo estudio, aquellos que piden ayuda tienden a recurrir a amigos y familiares antes que a servicios profesionales. Se hace evidente que un sistema basado únicamente en la intervención (y no en la prevención) no será capaz de cubrir las necesidades de la mayoría de los adolescentes implicados en relaciones de pareja violentas (Crooks et al., 2011).

En la actualidad, son numerosos los investigadores y políticos que insisten en la necesidad de invertir en 
programas de prevención primaria con niños y adolescentes con objeto de enseñarles habilidades relacionales saludables como parte fundamental de su desarrollo. Las escuelas se consideran el lugar ideal para desarrollar este tipo de programas. De los argumentos a favor de la prevención de la violencia en las relaciones de pareja basada en el trabajo con los adolescentes en el entorno escolar, destacan tres: la importancia de realizar prevención primaria o de carácter general, los beneficios que ofrece el entorno escolar para la implementación de programas de intervención, y que la adolescencia es una periodo de la vida especialmente apropiado para prevenir la implicación en relaciones íntimas violentas (Crooks et al., 2011). A pesar de la creciente popularidad de los programas de intervención desarrollados en el entorno escolar, todavía hay poca investigación empírica en este ámbito, y la mayoría de los programas implementados no se han evaluado de forma rigurosa, con grupos control con asignación aleatoria, así como con suficientes medidas y un seguimiento adecuado (Crooks et al., 2011).

\section{Programas de prevención de la violencia en las relaciones de pareja en la adolescencia: La Cuarta $R$}

En una revisión realizada por Whitaker y colaboradores en el 2006, únicamente se encontraron dos programas de prevención de la violencia en las relaciones de pareja durante la adolescencia que mostraran ser eficaces habiendo sido evaluados de forma rigurosa mediante un diseño con grupo control y asignación aleatoria. Uno de ellos, el Proyecto de Relaciones entre Jóvenes o Youth Relationships Project (Wolfe et al., 1996), es un programa de prevención para jóvenes en riesgo de ejercer violencia de pareja debido a un historial de maltrato infantil o por haber estado expuestos a violencia doméstica. El otro programa destacado por Whitaker y colaboradores (2006), Citas Seguras o Safe Dates (Foshee, Bauman, Ennett, Suchindran, Bene- field y Linder, 2005), es un programa de prevención de la violencia en las relaciones de pareja en la adolescencia creado para aplicarlo de forma generalizada en los colegios. Posteriormente, se ha publicado la evaluación de la efectividad (con grupo control y asignación aleatoria) de un tercer programa, la Cuarta $R$ o Fourth $R$ (Wolfe et al., 2009).

La Cuarta $R$ o Fourth $R$ es un programa de prevención primaria o universal creado para su aplicación en el entorno escolar, que tiene como objetivo prioritario la prevención de la violencia contra la pareja y de conductas de riesgo relacionadas con esta violencia y que está diseñado para incluir en la intervención a los estudiantes, los profesores, los padres y la comunidad. El elemento central de la Cuarta $R$ es un programa de 21 temas curriculares relativos a las habilidades que tratan de promover las relaciones saludables, y cuyo principal objetivo es la violencia, el comportamiento sexual de alto riesgo y el uso de sustancias entre los adolescentes (Crooks et al., 2011). La intervención está programada y diseñada para coincidir con los intereses y motivaciones naturales de los adolescentes para aprender acerca de cuestiones relacionadas con los estilos de vida, un factor esencial en la promoción de la salud de los jóvenes (Irwin et al., 2002). Los encargados de impartir el programa son los propios profesores, que reciben formación especializada. Esto supone una ventaja en términos económicos y garantiza la viabilidad de la intervención (Wolfe et al., 2009). El argumento central es que las habilidades para relacionarse pueden ser enseñadas de la misma manera que las otras "tres R" (la lectura, la escritura y la aritmética) y que deberían establecerse estas habilidades como parte fundamental del currículo de la educación secundaria, igual que el resto de habilidades. La Cuarta $R$ se compone de tres unidades centradas en la violencia, el consumo de sustancias y el comportamiento sexual saludable.

En conjunto, estas tres unidades tratan los tres tipos de conductas de riesgo que co-ocurren en los adolescentes y que determinan las relaciones de pareja entre

Tabla 1. Principales Características de Programas Efectivos de Prevención de Violencia en las Relaciones de Pareja entre Adolescentes

Proyecto de Relaciones entre Jóvenes (Wolfe et al., 1996)

Población Diana

Contexto/Profesionales

Duración
Jóvenes (14-17 años) con riesgo de ejercer violencia contra su pareja

Comunidad/Profesionales de los servicios sociales

18 sesiones

Citas Seguras (Foshee et al., 2005)

Población Diana

Contexto/Profesionales

Duración
Grados 7 a 10 (conjunto de sesiones comunes para todos los niveles)

Colegio-Instituto/Profesores con formación específica

9 sesiones

Cuarta R (Wolfe et al., 2009)

Población Diana

Contexto/Profesionales

Duración
Grados 7 a 12 (sesiones diferentes para cada grado)

Colegio-Instituto/ Profesores con formación específica

21 sesiones 
Tabla 2. Sesiones, Contenido y Ejemplos del Currículo de la Cuarta $R$

Unidad 1. Seguridad personal y prevención de lesiones

\begin{tabular}{lll}
\hline Sesión & Contenido & Ejemplos \\
\hline Sesión 1 & Relaciones saludables & Mito/realidad de las relaciones durante la adolescencia \\
Sesión 2 & Barreras para las relaciones saludables & Habilidades de escucha activa \\
Sesión 3 & Potenciadores de la violencia & Efectos grupales sobre la violencia \\
Sesión 4 & Conflicto y resolución de conflictos & Habilidades de comunicación \\
Sesión 5 & Violencia en los medios & Videos con ejemplos \\
Sesión 6 & Habilidades de resolución de conflictos & Derechos y responsabilidades cuando acaba una relación \\
Sesión 7 & Actuaciones en la escuela y la comunidad &
\end{tabular}

Unidad 2. Crecimiento y sexualidad saludable

\begin{tabular}{lll}
\hline Sesión & Contenido & Ejemplos \\
\hline Sesión 1 & Sexualidad saludable & Clarificación de mitos \\
Sesión 2 & Sexualidad en los medios & Presión para tener relaciones sexuales \\
Sesión 3 & Sexualidad responsable & Relaciones saludables \\
Sesión 4 & Prevención de embarazos no deseados y enfermedades de trasmisión sexual & \\
Sesión 5 & Asertividad para el manejo de la presión en las relaciones & Negociación, saber decir no \\
Sesión 6 & Sexualidad: responsabilidades y consecuencias & Abuso sexual \\
Sesión 7 & Toma de decisiones en la sexualidad y recursos comunitarios & Búsqueda de recursos comunitarios \\
\hline
\end{tabular}

Unidad 3. Uso y abuso de substancias

\begin{tabular}{|c|c|c|}
\hline Sesión & Contenido & Ejemplos \\
\hline Sesión 1 & Mitos/realidades y definiciones & $\begin{array}{l}\text { Dinámica para evaluar los valores y opiniones de los ado- } \\
\text { lescentes }\end{array}$ \\
\hline Sesión 2 & Efectos del uso y abuso de substancias & Debate sobre los efectos físicos y psicológicos \\
\hline Sesión 3 & Toma de decisiones sobre fumar & Debate: ¿por qué fuman los jóvenes? \\
\hline Sesión 4 & Factores que influyen la decisión de consumir sustancias & $\begin{array}{l}\text { Debate sobre la influencias de los medios, los iguales y la } \\
\text { cultura }\end{array}$ \\
\hline Sesión 5 & Adquisición de habilidades de rechazo ante la presión para el consumo de drogas & Negociación, saber decir no \\
\hline Sesión 6 & Practicar habilidades y recursos comunitarios & Ejercicios de role-playing \\
\hline Sesión 7 & Afrontar y reconocer las relaciones entre consumo de drogas, sexo y violencia & \\
\hline
\end{tabular}

los jóvenes. Los adolescentes practican mediante roleplaying diferentes formas de resolver conflictos, como participantes y como espectadores. Además, aprenden a aplicar los conocimientos en cada una de las tres áreas. Por ejemplo, en lugar de aprender la comunicación asertiva en general, aprenden a practicar la comunicación asertiva en situaciones realistas, como las citas y los conflictos entre iguales, la presión para consumir drogas o alcohol y la presión para tener relaciones sexuales (Crooks et al., 2011). Otros componentes del programa se centran en la comunidad escolar y los padres. La intervención en la escuela incluye la sensibilización y educación del personal y los profesores, información sobre el programa y actividades complementarias lideradas por estudiantes pertenecientes a los Comités de Acción Juvenil para aumentar los vínculos entre los compañeros. Cada uno de estos Comités se desarrolla con la orientación de un profesor, sobre la base de un manual que proporciona orientación en la contratación, formación, planificación y realización de actividades y que evalúa el éxito. A los padres se les proporciona información sobre estrate- gias relevantes para el desarrollo de los adolescentes. Además del programa básico (diseñado para estudiantes de noveno grado, con edades comprendidas entre los 14 y los 15 años), hay diversas ampliaciones del programa para adaptarlo a otros grados, otras áreas curriculares y para poblaciones especiales. Así, por ejemplo, los autores de la Cuarta $R$ han desarrollado una versión con perspectiva aborigen para respetar las diferencias culturales y el contexto histórico de los pueblos aborígenes en Canadá. Esta adaptación incluye modelos culturalmente relevantes de las relaciones saludables desde una perspectiva global y de maneras de lograr el apoyo de la familia extensa y la comunidad en la toma de decisiones saludables, elementos importante en las culturas aborígenes (Crooks et al., 2011).

\section{Evaluación de la efectividad del Programa la Cuarta $R$}

La eficacia de este programa ha sido evaluada siguiendo un diseño experimental con grupos control y 
asignación aleatoria con una muestra de 1722 estudiantes de 20 colegios, por Wolfe y colaboradores (2009). La evaluación se realizó en escuelas del suroeste de Ontario (Canadá). Los colegios que podían participar en la aplicación y posterior evaluación del programa fueron aquellos con estudiantes de población general y cuya dirección estaba de acuerdo con la realización de una asignación aleatoria, la formación del profesorado, desarrollar la intervención relativa a la condición experimental asignada, la evaluación y con restringir la aplicación de programas similares durante el tiempo de aplicación y evaluación de la Cuarta $R$. Todos los estudiantes matriculados en la asignatura "Salud y Educación Física" de noveno grado podían participar en la intervención y la evaluación.

\section{Diseño experimental e intervención}

Los colegios fueron estratificados en función del tamaño ( $\geq 500$ vs. $<500$ ) y tipo de localidad (rural vs. urbana). Los colegios fueron asignados aleatoriamente por estratos a la condición experimental o a la condición control. En la condición experimental se aplicaba la intervención denominada la "Cuarta R: Habilidades para las relaciones entre los jóvenes". Ésta se implementaba formando parte del currículo de una asignatura, de forma que no era necesario un tiempo adicional, ampliación de horarios o más recursos humanos. Los componentes del programa del nivel individual y escolar se diseñaron con objeto de integrar estrategias basadas en la evidencia para tratar la violencia en las relaciones de pareja en la adolescencia y las conductas de riesgo relacionadas, tales como estrategias de negociación, habilidades para decir que no, así como ejercicios para definir y ensayar responsabilidades asociadas con las relaciones saludables. Se proporcionaron ejemplos de conflictos entre iguales y entre parejas a las que se enfrentan los adolescentes, así como instrucciones para la realización de role-playings diseñados para incrementar habilidades sociales y de resolución de problemas.

La intervención en el nivel individual (estudiantes) consistió en 21 lecciones/clases de 75 minutos, detalladas en un manual y aplicadas por los profesores de Salud y Educación Física. Se proporcionaron esquemas detallados de cada uno de los temas o lecciones, recursos audiovisuales, ejercicios de role-playing y folletos. La intervención adoptó una perspectiva de género ante la violencia en las relaciones de pareja en la adolescencia, enfatizando los patrones y factores específicos de género y adecuando las actividades (Crooks, Wolfe y Jaffe, 2007).

En cuanto a los componentes de la intervención en el nivel escolar incluyeron formación adicional a los profesores en el ámbito de la violencia en las relaciones de noviazgo y las relaciones saludables, información a los padres y la creación de "comités escolares de salud" dirigidos por los estudiantes. Los profesores recibieron un seminario de formación de seis horas impartido por un educador y un psicólogo con el objetivo de familiarizarse y revisar los materiales y participar en ejercicios de potenciación de habilidades para los adolescentes. Los padres recibieron información en las sesiones de orientación del Grado 9 y a través de cuatro boletines que describían los temas que iban a ser tratados. Además, cada colegio recibió un manual titulado Escuelas Seguras para los Jóvenes (Youth Safe Schools), en donde se describen diversas maneras de implicar a los estudiantes en actividades escolares y comunitarias de prevención de la violencia.

La implicación de los profesores se consideraba el elemento central para garantizar el cumplimiento de los estándares del programa y la adherencia al mismo de los estudiantes. En este sentido, los profesores recibieron esquemas detallados de las lecciones a impartir, visionaron videos de entrenamiento y demostraciones a través de role-playings y recibieron feedback individualizado por parte de un educador experimentado. Además, los profesores que participaron en la evaluación implementaron el currículum al menos durante un semestre antes de la aplicación de las condiciones experimentales con objeto de familiarizarse con el mismo y garantizar su estandarización.

Los temas básicos que componen el currículo de la asignatura "Salud y Educación Física" para el Grado 9 en Ontario conformaron el contenido estándar impartido en los grupos control. Los profesores en los colegios control debían enseñar las 21 lecciones requeridas que cubren las mismas tres unidades que en los colegios incluidos en la condición experimental, pero sin entrenamiento ni formación especializada en estos temas, o acceso a un currículum estructurado que enfatice el desarrollo de las habilidades sociales y de relación para prevenir la violencia y las conductas de riesgo.

\section{Objetivos e instrumentos de medida de los resultados}

El objetivo principal de la investigación era determinar si un currículum que integrara la prevención de la violencia en las relaciones de pareja entre adolescentes con temas sobre relaciones saludables, salud sexual y consumo de sustancias, reduce la violencia en las relaciones de pareja entre adolescentes dos años después en mayor medida que el contenido académico estándar. Como objetivos secundarios se contemplaba la reducción de conductas de riesgo relacionadas tales como la violencia entre iguales, el consumo de sustancias y la conducta sexual de riesgo.

La variable resultado principal era el comportamiento violento en las relaciones de pareja entre adolescentes dos años después de establecida la línea base. Esta variable se evaluó con 8 ítems del Inventario de Relaciones Conflictivas en el Noviazgo Adolescente 
(Conflict in Adolescent Dating Relationships Inventory; Wolfe, Scott, Reitzel-Jaffe, Wekerle, Grasley y Straatman, 2001). Los estudiantes tenían que marcar las conductas (si o no) que hubieran realizado en el año anterior hacia su novio o novia "mientras teníais una discusión, estabais enfadados el uno con el otro o teniendo una pelea". Se consideraba que existía violencia en la relación si el estudiante marcaba 1 ó más ítems que implicaran abuso físico o amenaza de daño (e.g., "Yo le empujé o le zarandeé" o "Le amenacé con hacerle daño"). Los sujetos que no hubieran tenido pareja a lo largo del año anterior o que no hubieran marcado ningún ítem recibían la puntuación de 0 .

La evaluación de la violencia física entre iguales se basó en las respuestas a tres ítems que hacían referencia a los tres meses previos (" $i$ te has peleado con alguien, hasta el punto de que hayáis necesitado curar alguna herida?", “ ¿has participado en alguna pelea en la que hayas golpeado a alguien con otra cosa que no sean tus propias manos?” y "¿has pegado, abofeteado o dañado físicamente a otro adolescente con la intención de burlarte de él/ella o humillarlo/a?"), basados en el inventario de conductas delictivas de la Encuesta Nacional Longitudinal a Niños y Jóvenes (National Longitudinal Survey of Children and Youth; Human Resources Development Canada, 2001). La experiencia con el alcohol y las drogas ilegales fue evaluada utilizando la misma encuesta nacional, considerándose que existía un problema con el consumo de sustancias en base a la presencia de uno de los siguientes cuatro criterios: beber alcohol 1 ó 2 días a la semana o más, tomar 5 ó más bebidas alcohólicas a la vez en los últimos 30 días, consumir marihuana 1 ó 2 días a la semana o más, o haber probado cualquier otra droga ilegal en los tres meses pasados. La utilización del condón fue definida en términos de utilizarlo siempre que se mantuvieran relaciones sexuales únicamente para aquellos participantes sexualmente activos.

\section{Resultados}

De los 2243 estudiantes susceptibles de participar en el estudio de los 20 colegios implicados, 1722 (76.8\%) dieron su consentimiento. Los estudiantes que participaron en el estudio cumplimentaron una batería de cuestionarios al inicio y un cuestionario de seguimiento dos años y medio después. En la fase de seguimiento, el $88 \%$ de la muestra proporcionó los datos requeridos. Las comparaciones pre-test de los sujetos perdidos en el seguimiento $(n=202)$, con aquellos que sí que completaron el estudio, indican que los primeros era más probable que fueran varones $(60 \%)$ y que hubieran informado de tener problemas de consumo de alcohol en el pase de instrumentos realizado en la línea base, mientras cursaban el noveno grado (25\% vs. $16 \%$ respectivamente); no se encontraron diferencias en relación a la violencia en las relaciones de pareja entre los que participaron en el seguimiento y los que no lo hicieron.

Efectos de la intervención en la violencia contra la pareja en las relaciones entre adolescentes. Al finalizar el grado 11, la violencia contra la pareja se había incrementado del $1.1 \%$ cuando los estudiantes tenía 13-14 años, al 8.5\% cuando tenían 16-17 años. Además, este tipo de violencia era significativamente mayor entre los estudiantes que formaban parte del grupo control que entre los que formaban parte del grupo en el que se había desarrollado la intervención ( $9.8 \%$ vs. $7.4 \%$ respectivamente). Análisis posteriores de la violencia en las relaciones de pareja entre adolescentes mostraron un efecto significativo para la interacción entre las variables sexo $\times$ grupo, que indicaba que el efecto de la intervención difería significativamente entre chicos y chicas. Así, los chicos en los grupos de intervención era menos probable que se implicaran en conductas violentas hacia su pareja que los chicos de los grupos control $(2.7 \%$ vs. $7.1 \%)$. Sin embargo, las chicas tenían índices similares de conducta violenta hacia su pareja en ambos grupos (11.9\% vs. $12.0 \%$ respectivamente).

Efectos de la intervención en las variables resultado secundarias. Las diferencias entre los grupos control y los grupos experimentales no fueron significativas en relación a la violencia física entre iguales (17.1\% vs. $18.4 \%)$, consumo de sustancias $(47.8 \%$ vs. $52.4 \%$ ), o uso del condón $(54.1 \%$ vs. $55.0 \%)$. Sin embargo, los efectos de la intervención sobre el uso de condones presentaba diferencias significativas entre chicas y chicos. En concreto, la utilización de condones entre los chicos sexualmente activos de las escuelas en las que se realizó la intervención fue significativamente mayor $(67.9 \%)$ que en las escuelas control (58.6\%), y el uso del condón para sus parejas fue significativamente menor para las chicas en los grupos de intervención que en los grupos control (50.7\%).

\section{Consideraciones finales}

Los resultados de la evaluación de este programa de intervención avalaron la hipótesis de que la inclusión de contenidos curriculares sobre relaciones sociales saludables, así como diferentes formas de evitar situaciones de violencia de pareja en las relaciones entre adolescentes, permitía reducir este tipo de violencia en el tiempo ( 2.5 años después). No obstante, este efecto parece limitarse únicamente a los chicos. Por otro lado, diversos análisis exploratorios también indicaron que los chicos en las escuelas que participaron en este programa de intervención se involucraban con menor frecuencia en prácticas sexuales de riesgo (utilización del condón). Este resultado es potencialmente importante dada la evidencia empírica reciente que indica que la no utilización del condón es mayor entre chicos adolescentes involucrados en situaciones de violencia de 
pareja, en particular en el contexto de relaciones estables (Raj, Reed, Miller, Decker, Rothman y Silverman, 2007).

Con respecto a los resultados obtenidos con las chicas, es importante subrayar que los indicadores utilizados para evaluar la violencia consistían en informar acerca de la realización o no de un acto específico, como pegar, empujar o amenazar a la pareja. Estos indicadores, sin embargo, no incorporan las motivaciones o circunstancias que pueden rodear a un acto de violencia, ni permiten distinguir entre actos de agresión o de defensa. Esto podría explicar el hecho de que las chicas señalaran mayores tasas de violencia que los chicos (Foshee y Matthew, 2007). Así mismo, y con respecto a las tasas más elevadas de violencia de las chicas y su menor respuesta a la intervención, hay varias cuestiones que merecen ser consideradas. Generalmente ambos sexos muestran una mayor aceptabilidad del uso de la violencia física para las chicas que para los chicos y muchos perciben un doble rasero con respecto a la respuesta de los adultos ante la violencia física de los chicos en comparación con la de las chicas. Además, las chicas tienden a describir las circunstancias que rodean su uso de la violencia como una respuesta a la violencia de sus parejas (Price y Byers, 1999; Sears, Byers, Whelan y Saint-Pierre, 2006). Por otro lado, las chicas víctimas de violencia tienen una mayor probabilidad que los chicos de experimentar miedo, ansiedad y daños, así como de expresar el deseo de abandonar la situación como una medida de autoprotección (Follingstad, Wright, Lloyd y Sebastian, 1991; Watson, Cascardi, Avery-Leaf y O'Leary, 2001), algo que ocurre de forma similar en las situaciones de violencia de pareja entre adultos (Krug, Dahlberg, Mercy, Zwi y Lozano, 2002).

Estas consideraciones subrayan la importancia que tiene comprender el contexto en el que estas conductas de violencia ocurren para desarrollar programas de intervención efectivos. En este sentido, sería deseable poseer mayor información contextual acerca de la naturaleza de la violencia que experimenta cada sexo para poder desarrollar intervenciones con objetivos más precisos. En definitiva, la evaluación del programa de prevención la Cuarta $R$ demuestra que los profesores con una formación suplementaria pueden implementar programas de prevención basados en la evidencia científica con una suficiente fidelidad y efectividad que permitan obtener mejoras significativas en comparación con otros acercamientos de intervención en el aula.

\section{Referencias}

Arriaga, X. B. y Foshee, V. A. (2004). Adolescent dating violence: Do adolescente follow in their friends', or their parents', footsteps? Journal of Interpersonal Violence, 19, 162-184.
Ashley, O. y Foshee, V. (2005). Adolescent help-seeking for dating violence: Prevalence, sociodemographic correlates, and sources of help. Journal of Adolescent Health, $36,25-31$.

Coker, A. L., McKeown, R. E., Sanderson, M., Davis, K. E., Valois, R. F. y Huebner, E. S. (2002). Severe dating violence and quality of life among South Carolina high school students. American Journal of Preventive Medicine, 19, 220-227.

Conolly, J., Furman, W. y Konarski, R. (2000). The role of peers in the emergence of heterosexual romatic relationships in adolescence. Child Development, 71, 1395-1408.

Crooks, C. V., Jaffe, P. G., Wolfe, D. A., Hughes, R. y Chiodo, D. (2011). School-based dating violence prevention. From single events to evaluated, integrated programming. En C. M. Renzetti, J. L. Edleson y R. K. Bergen (Eds.), Sourcebook on violence against women (pp. 327-349). London: Sage.

Crooks, C. V., Wolfe, D. A. y Jaffe, P. G. (2007). Schoolbased adolescent dating violence prevention: enhancing effective practice with a gender strategic approach. En K. Kendall-Tackett y S. Giacomoni (Eds.), Intimate partner violence. Kingston, NJ: Civic Research Institute.

Centers for Disease Control and Prevention (2006). Physical dating violence among high school students - United States 2003. Morbidity and Mortality Weekly Report, 55, 532-535.

Eaton, D. K., Kann, L., Kinchen, S., Ross, J., Hawkins, J., ... Weschsler, H. (2006). Youth risk behavior surveillance United States, 2005. Morbidity and Mortality Weekly Report Surveillance Summaries, 55, 1-108.

Follingstad, D. R., Wright, S., Lloyd, S. y Sebastian, J. A. (1991). Sex differences in motivations and effects in dating violence. Family Relations, 40, 51-57.

Foshee, V. A., Bauman, K. E. y Linder, G. F. (1999). Family violence and the preparation of adolescent dating violence: Examining social learning and social control processes. Journal of Marriage and the Family, 61, 331-342.

Foshee, V. A., Bauman, K. E., Ennett, S. T., Suchindran, C., Benefield, T. y Linder, G. F. (2005). Assessing the effects of the dating violence prevention program "Safe Dates" using random coefficient regression modeling. Prevention Science, 6, 245-258.

Foshee, V. A. y Matthew, R. A. (2007). Adolescent dating abuse perpetration: A review of findings, methodological limitations, and suggestions for future research. En D. J. Flannery, A. T. Vazonsyi y I. D. Waldman (Eds.), The Cambridge handbook of violent behavior and aggression (pp. 431-449). New York: Cambridge University Press.

Grunbaum, J. A., Kann, L., Kinchen, S. A., Williams, B., Ross, J. G., ... Collins, J. (2001). Youth risk behavior surveillance - United States, 2001. Morbidity and Mortality Weekly Report Surveillance Summaries, 51, 1-62.

Hahn, R., Fuqua-Whitley, D., Wethington, H., Lowy, J., Liberman, A., ... Dahlberg, L. (2007). The effectiveness of universal school-based programs for the prevention of violent and aggressive behavior: A report on recommendations of the Task Force on Community Preventive 
Services. Morbidity and Mortality Weekly Report Recommendations Repport, 56, 1-12.

Halpern, C. T., Spriggs, A. L., Martin, S. L. y Kupper, L. L. (2009). Patterns of intimate partner violence victimization form adolescence to young adulthood in a nationally representative sample. Journal of Adolescent Health, 45, 508-516.

Human Resources Development Canada (2001). National longitudinal survey of children and youth: Cycle 4 20002001. Otawa, ON: Human Resources Development Canada.

Irwin, C. E. Jr., Burg, S. J. y Uhler Cart, C. (2002). America's adolescents: where have we been, where are we going? Journal of Adolescent Health, 31, 91-121.

Krug, E. G., Dahlberg, L. L., Mercy, J. A., Zwi, A. B. y Lozano, R. (2002). World report on violence and health. Geneva, Switzerland: World Health Organization.

Lewis, S. F. y Fremouw, W. (2001). Dating violence: a critical review of the literature. Clinical Psychology Review, 21, 105-127.

Malik, S., Sorenson, S. B. y Aneshensel, C. A. (1997). Community and dating violence among adolescents: Perpetration and victimization. Journal of Adolescent Health, 21, 291-302.

Miller, J. y White, N. A. (2003). Gender and adolescent relationship violence: A contextual examination. Crimino$\log y, 41,1207-1248$.

Odgers, C. L. y Moretti, M. M. (2002). Aggressive and antisocial girls: research update and challenges. International Journal of Forensic Mental Health, 1, 103-119.

O'Keefe, M. (2005). Teen dating violence: A review of risk factors and prevention efforts. VAWNet Applied Research Forum. Extraído el 15 de Mayo de 2009 de http://new.vawnet.org/Assoc_Files_VAWnet/AR_TeenD atingViolence.pdf

Price, E. L. y Byers, E. S. (1999). The attitudes towards dating violence scales: development and initial validation. Journal of Family Violence, 14, 351-375.

Raj, A., Reed, E., Miller, E., Decker, M. R., Rothman, E. F. y Silverman, J. G. (2007). Contexts of condom use and non-condom use among young adolescent male perpetrators of dating violence. AIDS Care, 19, 970-973.

Sears, H. A, Byers, E. S., Whelan, J. J. y Saint-Pierre, M. (2006). Dating Violence Research Team. "If it hurts you, then it is not a joke": adolescents' ideas about girls' and boys' use and experience of abusive behavior in dating relationships. Journal of Interpersonal Violence, 21, 1191-1207.

Silverman, J. G., Raj, A., Mucci, L. A. y Hathaway, J. E. (2001). Dating violence against adolescent girls and associated substance use, unhealthy weight control, sexual risk behavior, pregnancy, and suicidality. Journal of the American Medical Association, 286, 572-579.

Swahn, M. H., Simon, T. R., Hertz, M. F., Arias, I., Bossarte, R. M., ... Hamburger, M. E. (2008). Linking dating vio- lence, peer violence, and suicidal behaviors among highrisk youth. American Journal of Preventive Medicine, 34, 30-38.

US Department of Health and Human Services (2000). Healthy people 2010: Understanding and improving health. Washington, DC: Autor.

Watson, J. M., Cascardi, M., Avery-Leaf, S. y O'Leary, K. D. (2001). High school students' responses to dating aggression. Violence and Victims, 16, 339-348.

Wekerle, C. y Wolfe, D. A. (1999). Dating violence in midadolescence: Theory, significance and emerging prevention initiatives. Clinical Psychology Review, 19, 435-456.

Whitaker, D. J., Morrison, S., Lindquist, C., Hawkins, S. R., O’Neil, J. A., ... Reese, L. R. (2006). A critical review of interventions for the primary prevention of perpetration of partner violence. Aggresion and Violent Behavior, 11, 151-166.

Wolfe, D. A., Crooks, C., Jaffe, P., Chiodo, D., Hughes, R., Ellis, W., ... Donner, A. (2009). A school-based program to prevent adolescent dating violence. A cluster randomized trial. Archives of Pediatrics \& Adolescent Medicine, 163, 692-699.

Wolfe, D. A., Crooks, C. C., Chiodo, D. y Jaffe, P. (2009). Child maltreatment, bullying, gender-based harassment, and adolescent dating violence: Making the connections. Psychology of Women Quarterly, 33, 21-24.

Wolfe, D. A., Jaffe, P. G. y Crooks, C. V. (2006). Adolescent risk behaviors: why teens experiment and strategies to keep them safe. New Haven CT.: Yale University Press.

Wolfe, D. A., Scott, K. L. y Crooks, C. V. (2005). Abuse and violence in adolescent girls' dating relationships. En D. J. Bell, S. L. Foster y E. J. Mash (Eds.), Handbook of behavioral and emocional problems in girls: Issues in clinical chils psychology (pp. 381-414). Nueva York: Kluwer Academic/Plenum Publishers.

Wolfe, D. A., Scott, K. L., Reitzel-Jaffe, D., Wekerle, C., Grasley, C. y Straatman, A. (2001). Development and validation of the Conflict in Adolescent Dating Relationships Inventory. Psychological Assessment, 13, 277-293.

Wolfe, D. A., Scott, K. L., Wekerle, C. y Pittman, A. L. (2001). Child maltreatment: Risk of adjustment problems and dating violence in adolescence. Journal of the American Academy of Child and Adolescent Psychiatry, 40, 282-289.

Wolfe, D. A., Wekerle, C., Gough, R., Reitzel-Jaffe, D., Grasley, C., ... Stumpf, J. (1996). The youth relationships manual: A group approach with adolescents for the prevention of woman abuse and the promotion of healthy relationships. Thousand Oaks, CA: Sage.

Wolitzky-Taylor, K. B., Ruggiero, K. J., Danielson, C. K., Resnick, H. S., Hanson, R. F., ... Kilpatrick, D. G. (2008). Prevalence and correlates of dating violence in a national simple of adolescents. Journal of the American Academy of Child and Adolescent Psychiatry, 47, 755-762.

Manuscrito Recibido: 22/04/2011 Revisión Recibida: 17/06/2011 Manuscrito Aceptado: 17/06/2011 